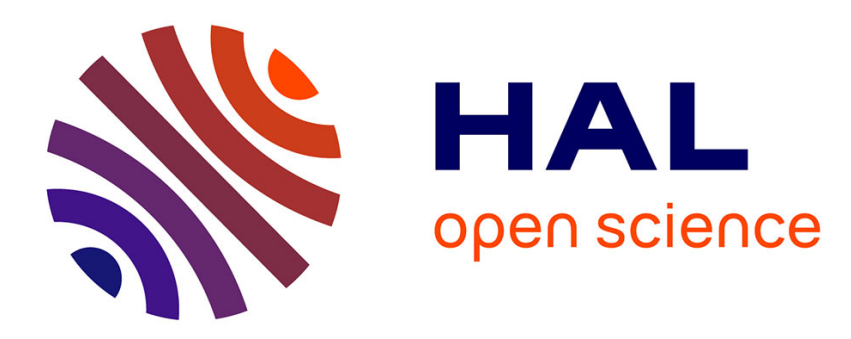

\title{
Approximations fonctionnelles des courbes des espaces projectifs
}

Patrice Philippon

\section{To cite this version:}

Patrice Philippon. Approximations fonctionnelles des courbes des espaces projectifs. 2007. hal00480924

\section{HAL Id: hal-00480924 \\ https://hal.science/hal-00480924}

Preprint submitted on 5 May 2010

HAL is a multi-disciplinary open access archive for the deposit and dissemination of scientific research documents, whether they are published or not. The documents may come from teaching and research institutions in France or abroad, or from public or private research centers.
L'archive ouverte pluridisciplinaire HAL, est destinée au dépôt et à la diffusion de documents scientifiques de niveau recherche, publiés ou non, émanant des établissements d'enseignement et de recherche français ou étrangers, des laboratoires publics ou privés. 


\title{
Approximations fonctionnelles des courbes des espaces projectifs
}

\author{
Patrice PHILIPPON \\ UMR 7586 du CNRS - Géométrie et Dynamique, \\ Université P. \& M. Curie, T.46-56, 5ème ét., F-75252 PARIS cedex 05.
}

\section{$\S 1$. Le contexte}

Ravivant une idée de A. Durand [7], divers auteurs, D. Roy et M. Waldschmidt [24], [25], M. Waldschmidt [26], [27], [28], M.Laurent [11], [12], M. Laurent et D. Roy [14], [15] et l'auteur [20], [21], chap. 4, ont montré que les mesures d'approximation par les nombres algébriques sont susceptibles de fournir des résultats d'indépendance algébrique, pourvu qu'on sache déterminer la qualité d'approximation «générique » d'un uplet de nombres.

En effet, on peut munir l'ensemble des sous-corps du corps des nombres complexes $C$ algébriquement clos, de degrés de transcendance finis, d'une distance pour laquelle tout corps de degré de transcendance $\leq t$ devrait conjecturalement être à distance $\leq \frac{t}{t+1}$ du corps des nombres algébriques. D'un autre côté, une démonstration de transcendance effective fournit une mesure d'approximation par les nombres algébriques, c'est-à-dire une minoration de la distance du corps de degré de transcendance fini $t$ étudié au corps des nombres algébriques, et la comparaison avec la majoration de la distance par $\frac{t}{t+1}$ donne une minoration de $t$, les rayons critiques étant $\frac{1}{2}, \frac{2}{3}, \frac{3}{4}, \frac{4}{5}, \ldots$ Il est remarquable que cette approche de l'indépendance algébrique repose sur les mêmes principes que celle via les critères pour l'indépendance algébrique et devrait donc en offrir une alternative beaucoup plus maniable, car dégagée des hypothèses les plus contraignantes de ces critères.

Essentiellement deux types de conjectures ont été formulées pour l'approximation par les nombres algébriques. Partant de la dimension 1 ( $c f$. [24]) et se rattachant à un résultat de E. Wirsing [32], D. Roy et M. Waldschmidt demandent dans [25], conjecture 1.7, que tout $t$-uplet de nombres complexes puisse être approché par une infinité de $t$-uplets de nombres algébriques de degré et hauteur logarithmique absolue bornés à l'avance et avec un ordre d'approximation égal au produit de la borne imposée à la hauteur par la puissance $1+1 / t$ de celle imposée au degré.

Cette conjecture, trop optimiste, est infirmée par D. Roy dans [23] pour faire place à une autre aussi formulée par M. Laurent [11], [12] et M. Waldschmidt [29], [30], qui, 
elle, s'apparente à des travaux de G.V. Chudnovsky [5], chapitre 4. La différence avec [25] réside dans le fait qu'on y demande un contrôle de la hauteur du $t$-uplet de nombres algébriques relative à son corps de définition, plutôt que de la hauteur absolue.

D'un autre point de vue, nous avons formulé dans [20] des conjectures d'approximation plus souple quant au contrôle de l'approximation, que nous avons établies en petite dimension ou codimension dans [22]. Notre approche consiste à remarquer que la descente dans la démonstration des critères pour l'indépendance algébrique ( $c f$. l'auteur [19], [21], chap. 8, E.M. Jabbouri [9], C. Jadot [10]) peut être effectuée à l'aide de formes auxiliaires construites à cette unique fin, plutôt que dérivant d'une fonction auxiliaire éventuellement transcendante spécifique à un certain contexte.

Donnons un exemple du type de résultat souhaité. On désigne par $h(\cdot)$ la hauteur projective sur $\boldsymbol{P}_{n}(\overline{\boldsymbol{Q}})$ et Dist la distance projective sur $\boldsymbol{P}_{n}(\boldsymbol{C}) \quad(c f$. [22] pour ces notations et [20], problèmes $7 \mathrm{a}$ et $10 \mathrm{a}$, pour l'énoncé de la conjecture).

Conjecture - Il existe un réel $c_{n} \geq 1$ tel que pour tout $x \in \boldsymbol{P}_{n}(\boldsymbol{C})$ et tous $H, D$ réels assez grands il existe un cycle $Z \subset \boldsymbol{P}_{n}(\overline{\boldsymbol{Q}})$ de dimension 0 et défini sur $\boldsymbol{Q}$, satisfaisant $\operatorname{deg}(Z) \leq D^{n}, h(Z) \leq(H+D) D^{n-1}$ et

$$
\sum_{\alpha \in Z} \log \operatorname{Dist}(x, \alpha) \leq-c_{n}^{-1}(h(Z) D+\operatorname{deg}(Z) H)
$$

Le cas de dimension 1 (approximation d'un nombre complexe ou d'un point sur une courbe algébrique) est bien étudié et les résultats obtenus, avec une large palette de contrôle des degrés et hauteurs, offrent de bonnes qualités numériques (voir Y. Bugeaud [1], [2], G. Diaz [6], M. Laurent [13], M. Laurent et D. Roy [14], D. Roy et M. Waldschmidt [24] et E. Wirsing [32]). Toutefois, la versatilité des approximations en dimension 1 ne se propage pas en dimension supérieure où des variétés de dimension $>0$ peuvent passer très près d'un point sans qu'aucun de ses points algébriques de hauteur « générique» ne soit aussi proche de ce même point. C'est là, du reste, la source des «hypothèses techniques» qui fleurissent dans certains résultats d'indépendance algébrique de plus de deux valeurs de la fonction exponentielle, par exemple.

En dimension $>1$, nous avons démontré dans [22], les conjecture de [20] en nous limitant à la dimension $\leq 3$ ou à la codimension $\leq 3$ selon les types d'énoncés. D'un autre côté, M. Laurent et D. Roy [16] ont élaboré en dimension supérieure un critère pour l'indépendance algébrique avec multiplicité [15], ils en déduisent un résultat d'approximation en codimension 1 . Pour les applications de ces énoncés, on renvoie à notre texte [20] et on peut citer M. Waldschmidt [31], chap. 15, où il est expliqué comment la conjecture 15.31 de cette référence (à laquelle devrait pouvoir être substituée la conjecture ci-dessus) se combine à une autre conjecture 14.25 de mesure d'approximation simultanée de logarithmes de nombres algébriques, pour démontrer 
l'indépendance algébrique de logarithmes de nombres algébriques linéairement indépendants sur $\boldsymbol{Q}$.

Notre propos est ici autre, il s'agit de démontrer l'analogue fonctionnel des problèmes $7 \mathrm{a}$ et 10a que nous avons formulés dans [20] et de montrer en toutes dimensions les analogues fonctionnels des énoncés que nous avons établis dans [22] (voir théorèmes 1 et 7 des paragraphes 2 et 5 respectivement). Comme corollaire on établit un lemme de transfert entre mesures d'approximation et mesures d'indépendance algébrique (voir corollaire 4), analogue à celui de [22]. Nous démontrons également au paragraphe 6 des versions multi-projectives du théorème 1 et du lemme de transfert, qui donnent une meilleure idée du parallélisme avec le cas arithmétique, voir théorème 10 et corollaire 11. Ces derniers énoncés trouvent une application directe dans l'étude des lemmes de multiplicité tels que développés par E.Zorin [33], par exemple.

Ce travail se base uniquement sur les outils développés dans [22], qui découlent de la minoration de fonction de Hilbert géométrique de M. Chardin et l'auteur [4] et de la majoration de cette même fonction par M. Chardin [3]. Le seul obstacle existant pour démontrer en dimension $>3$ les versions arithmétiques des résultats établis ici (i.e. la conjecture énoncée plus haut), est l'obtention d'une minoration effective de la fonction de Hilbert arithmétique d'une variété projective définie sur $\boldsymbol{Q}$, parallèle aux résultats de [4] sur les fonctions de Hilbert géométriques.

\section{$\S 2$. Notations et résultats}

Soit $n \in \boldsymbol{N}^{*}, \boldsymbol{k}$ un corps commutatif, algébriquement clos, $z$ une variable sur $\boldsymbol{k}, C:=\boldsymbol{k}((z))$ et $\bar{C}$ une clôture algébrique de $C$. On note $\boldsymbol{P}_{n}$ l'espace projectif de dimension $n, d(X)$ le degré d'une sous-variété projective $X$ de $\boldsymbol{P}_{n}(\boldsymbol{k})$ et $c_{n}:=$ $4^{n^{2}+n+1} n$ ! . Pour $\phi \in \boldsymbol{P}_{n}(C)$ et $X$ une sous-variété projective de $\boldsymbol{P}_{n}(\boldsymbol{k})$ on note ${ }^{\dagger}$ $\operatorname{Ord}_{\phi}(X)$ la valuation de $X$ en $\phi$, déduite de la valuation $z$-adique de $C$ via la forme de Chow $f_{X}$ de $X$ (i.e. $\operatorname{Ord}_{\phi}(X):=\operatorname{ord}_{z}\left(\mathfrak{d}_{\phi}\left(f_{X}\right)\right)-(\operatorname{dim}(X)+1) \operatorname{deg}(X) \operatorname{ord}_{z}(\phi)$, où $\left.\operatorname{ord}_{z}(\phi):=\min _{0 \leq i \leq n} \operatorname{ord}_{z} \phi_{i}\right)$.

Remarque - On vérifie que la seule variété $Z$ de dimension 0 qui satisfait $\operatorname{Ord}_{\phi}(Z)>0$ est le point $\{\phi(0)\}$, pour laquelle on a en fait $\operatorname{Ord}_{\phi}(Z)=\operatorname{Ord}_{z}(\phi \wedge \phi(0))$.

$$
\begin{aligned}
& \text { Pour } \alpha, \beta \in \boldsymbol{P}_{n}(\bar{C}) \text { on note } \\
& \qquad \operatorname{Ord}_{z}(\alpha \wedge \beta):=\min _{0 \leq i<j \leq n} \operatorname{ord}_{z}\left(\alpha_{i} \beta_{j}-\alpha_{j} \beta_{i}\right)-\operatorname{ord}_{z}(\alpha)-\operatorname{ord}_{z}(\beta),
\end{aligned}
$$

qui mesure (le logarithme de l'inverse de) la «distance projective» induite par la valuation $z$-adique de $C$, entre les points $\alpha$ et $\beta$.

\footnotetext{
$\dagger$ Voir aussi le $\S 6$ pour plus de détails sur ces notations dans le cas multiprojectif.
} 
Si $\alpha \in \boldsymbol{P}_{n}(\overline{\boldsymbol{k}(z)})$ et $\alpha_{1}, \ldots, \alpha_{D}$ désignent ses conjugués sur $\boldsymbol{k}(z)$, on définit la hauteur du cycle $Z:=\left\{\alpha_{1}, \ldots, \alpha_{D}\right\}$ par la formule

$$
h(Z):=-\sum_{u \in \boldsymbol{P}_{1}(\overline{\boldsymbol{k}})} \operatorname{ord}_{z_{u}}\left(\prod_{i=1}^{D} \sum_{j=0}^{n} U_{j} \alpha_{i, j}\right),
$$

où on a posé $z_{u}=z-v$ si $u=(1: v)$ et $z_{u}=1 / z$ si $u=(0: 1)$ et $U_{0}, \ldots, U_{n}$ désignent des variables indépendantes sur $\overline{\boldsymbol{k}(z)}$. On notera que la forme $\prod_{i=1}^{D} \sum_{j=0}^{n} U_{j} \alpha_{i, j}$ appartient à $\boldsymbol{k}(z)\left[U_{0}, \ldots, U_{n}\right]$, est de degré $D$ et son ordre relatif à chaque paramètre $z_{u}$ est le minimum des ordres de ses coefficients.

On pose les définitions suivantes :

Définition - Soit $\delta \in \boldsymbol{N}^{*}$ et $X \subset \boldsymbol{P}_{n}(\boldsymbol{k})$ une variété projective, on dira qu'une sousvariété de $X$ est incomplètement définie sur $\boldsymbol{k}$ dans $X$ par des formes de degrés $\leq \delta$ si et seulement si elle est composante isolée de l'intersection de $X$ et d'hypersurfaces de $\boldsymbol{P}_{n}(\boldsymbol{k})$ de degrés $\leq \delta$.

Voici dans ce contexte notre résultat principal. On en donne une version multiprojective au paragraphe 6 .

Théorème 1 - Soit $\phi \in \boldsymbol{P}_{n}(C), 1 \leq d \leq n$ et $\delta \geq 2(n+1)$ un entier, alors si $X_{0}$ est une variété de dimension $d_{0} \geq d$ définie par des formes de degrés $\leq \delta$ et satisfaisant $\operatorname{Ord}_{\phi}\left(X_{0}\right)>c_{n}^{-1} \operatorname{deg}\left(X_{0}\right) \delta^{d_{0}}$, il existe une variété $X$ de dimension $d$, contenue dans $X_{0}$, telle que $\operatorname{deg}(X) \leq \operatorname{deg}\left(X_{0}\right) \delta^{d_{0}-d} \leq \delta^{n-d}$ et $\operatorname{Ord}_{\phi}(X)>c_{n}^{-1} \operatorname{deg}(X) \delta^{d}$.

Comme application nous produisons des cycles de points définis sur $\boldsymbol{k}(z)$ et approchant très bien $\phi$.

Corollaire 2 - Sous les hypothèses du théorème 1 avec $d=1$, si $\phi_{0} \neq 0$ et $\frac{\phi_{1}}{\phi_{0}} \in \boldsymbol{k}(z) \backslash \boldsymbol{k}$ il existe un cycle $Z \subset X_{0}(\overline{\boldsymbol{k}(z)})$ de dimension 0 et défini sur $\boldsymbol{k}(z)$, tel que $h(Z) \leq h\left(\phi_{0}: \phi_{1}\right) \operatorname{deg}\left(X_{0}\right) \delta^{d_{0}-1}, \operatorname{deg}(Z) \leq \operatorname{deg}\left(X_{0}\right) \delta^{d_{0}-1}$ et

$$
\sum_{\alpha \in Z} \operatorname{Ord}_{z}(\alpha \wedge \phi)>\left(2 c_{n}\right)^{-1}\left(\frac{h(Z)}{h\left(\phi_{0}: \phi_{1}\right)}+\operatorname{deg}(Z)\right) \delta
$$

En particulier, si $X_{0}$ est de codimension 1 le corollaire 2 permet d'établir : 
Lemme de transfert - Soit $b \geq 2^{n-1}, \mu \geq n \geq 2$ et $\phi \in \boldsymbol{P}_{n}(C)$ tel que $\phi_{0} \neq 0$ et $\frac{\phi_{1}}{\phi_{0}} \in \boldsymbol{k}(z) \backslash \boldsymbol{k}$, si une forme $P \in \boldsymbol{k}\left[x_{0}, \ldots, x_{n}\right]$, non nulle, satisfait $d^{\circ} P \geq 2(n+1)$ et

$$
\operatorname{ord}_{z}(P \circ \phi)-d^{\circ} P . \operatorname{ord}_{z}(\phi)>b d^{\circ} P^{\mu}
$$

alors il existe un cycle $Z \subset \boldsymbol{P}_{n}(\overline{\boldsymbol{k}(z)})$ de dimension 0 et défini sur $\boldsymbol{k}(z)$, contenu dans le lieu des zéros de $P$, satisfaisant

$$
\operatorname{deg}(Z) \leq b^{\frac{n-2}{n-1}} d^{\circ} P^{\frac{1+\mu(n-2)}{n-1}}, \quad h(Z) \leq h\left(\phi_{0}: \phi_{1}\right) b^{\frac{n-2}{n-1}} d^{\circ} P^{\frac{1+\mu(n-2)}{n-1}}
$$

et

$$
\begin{aligned}
\sum_{\alpha \in Z} \operatorname{Ord}_{z}(\alpha \wedge \phi) & >\frac{1}{4 c_{n}} b^{\frac{1}{n-1}}\left(\frac{h(Z)}{h\left(\phi_{0}: \phi_{1}\right)}+\operatorname{deg}(Z)\right) d^{\circ} P^{\frac{\mu-1}{n-1}} \\
& >\frac{1}{2 c_{n}} b^{\frac{1}{1+\mu(n-2)}}\left(\frac{1}{2}\left(\frac{h(Z)}{h\left(\phi_{0}: \phi_{1}\right)}+\operatorname{deg}(Z)\right)\right)^{\frac{\mu(n-1)}{1+\mu(n-2)}}
\end{aligned}
$$

\section{$\S 3$. La machinerie}

Le premier lemme, repris de [22], est la clef de la démonstration du théorème 1. Pour $Y \subset \boldsymbol{P}_{n}(\boldsymbol{k})$ un ensemble algébrique défini sur $\boldsymbol{k}$, on note $H_{g}(Y ; \delta)$ la valeur en $\delta$ de la fonction de Hilbert (géométrique) de $Y$, i.e. la dimension sur $\boldsymbol{k}$ de l'espace des formes à coefficients dans $\boldsymbol{k}$ en $x_{0}, \ldots, x_{n}$ de degré $\delta$ modulo l'idéal des formes s'annulant sur $Y$.

Lemme 3 - Soit $\delta \in \boldsymbol{N}^{*}$ et $Y \subset \boldsymbol{P}_{n}(\boldsymbol{k})$ une variété projective incomplètement définie par des formes de degrés $\leq \delta$, alors pour tout entier $\delta^{\prime} \geq 2(n+1)$ on a

$$
2 c_{n}^{-1} \min \left(1 ; \frac{\delta^{\prime}}{\delta}\right)^{\operatorname{codim}(Y)} \operatorname{deg}(Y) \delta^{\prime \operatorname{dim}(Y)}<H_{g}\left(Y ; \delta^{\prime}\right) \leq \operatorname{deg}(Y) \delta^{\prime \operatorname{dim}(Y)}+1
$$

Démonstration - Cela résulte des théorème 5 et corollaire 6 de [22] et de la valeur fixée de $c_{n}=4^{n^{2}+n+1} n$ !, la majoration est due à M. Chardin [3]. On notera que $Y$ est incomplètement définie par des formes de degré $\leq \delta$, son idéal de définition $I \subset \boldsymbol{k}[\boldsymbol{x}]$ contient une suite $q_{1}, \ldots, q_{\operatorname{codim}(Y)}$ de formes de degré $\leq \delta$, régulière dans l'anneau localisé $\boldsymbol{k}[\boldsymbol{x}]_{I}$.

Proposition 4 - Soit $c \geq 1$ un réel, $1 \leq d<n$ un entier, $\delta \in \boldsymbol{N}^{*}$ et $Y \subset \boldsymbol{P}_{n}(\boldsymbol{k})$ une variété projective contenant une sous-variété $Z$ de dimension $>d$ maximale satisfaisant $\operatorname{Ord}_{\phi}(Z)>c^{-1} \operatorname{deg}(Z) \delta^{\operatorname{dim}(Z)}$.

Alors, si $H_{g}(Y ; \delta)>2 c^{-1} \operatorname{deg}(Y) \delta^{\operatorname{dim}(Y)}$ il existe une sous-variété $Z^{\prime} \subset Y$ de dimension $\geq d$ telle que $H_{g}\left(Z^{\prime} ; \delta\right)<H_{g}(Y ; \delta), \operatorname{deg}\left(Z^{\prime}\right) \leq \operatorname{deg}(Z) \cdot \delta^{\operatorname{dim}(Z)-\operatorname{dim}\left(Z^{\prime}\right)}$ et $\operatorname{Ord}_{\phi}\left(Z^{\prime}\right)>c^{-1} \operatorname{deg}\left(Z^{\prime}\right) \delta^{\operatorname{dim}\left(Z^{\prime}\right)}$. 
Démonstration - Si $H_{g}(Z ; \delta)=H_{g}(Y ; \delta)$, on construit une hypersurface $H$ de degré $\delta$ ne contenant pas $Z$ telle que $\operatorname{Ord}_{\phi}(H)>c^{-1} \operatorname{deg}(Y) \cdot \delta^{\operatorname{dim}(Y)}$. La condition $\operatorname{ord}_{\phi}(H)>T$ s'exprime au travers de $[T]+1$ équations linéaires en les coefficients d'une équation de $H$, il s'agit donc simplement de résoudre un système linéaire en $H_{g}(Y ; \delta)$ inconnues et $\leq 2 c^{-1} \operatorname{deg}(Y) \cdot \delta^{\operatorname{dim}(Y)}$ équations ce qui est loisible grâce à l'hypothèse $H_{g}(Y ; \delta)>2 c^{-1} \operatorname{deg}(Y) . \delta^{\operatorname{dim}(Y)}$. Deux cas se présentent alors :

- $\max _{\alpha \in Y(\bar{C})} \operatorname{Ord}_{z}(\alpha \wedge \phi)>c^{-1} \operatorname{deg}(Y) \cdot \delta^{\operatorname{dim}(Y)}$ : dans ce cas on vérifie $Y=Z$ car

$$
\operatorname{Ord}_{\phi}(Y) \geq \max _{\alpha \in Y(\bar{C})} \operatorname{Ord}_{z}(\alpha \wedge \phi)>c^{-1} \operatorname{deg}(Y) \delta^{\operatorname{dim}(Y)}
$$

et $Z \subset Y$ est de dimension maximale satisfaisant cette inégalité. D'après les théorèmes de Bézout (voir [17], chap. 3, prop 4.11, avec $h(Y)=h(H)=\nu=0$ ) on a $\operatorname{deg}(Y \cdot H) \leq \operatorname{deg}(Y) . \delta \leq \delta^{\operatorname{codim}(Y \cdot H)}$ et

$$
\begin{aligned}
\operatorname{Ord}_{\phi}(Y \cdot H) & \geq \min \left(\operatorname{Ord}_{\phi}(Y) ; \operatorname{Ord}_{\phi}(H)\right) \\
& >c^{-1} \operatorname{deg}(Y) \cdot \delta^{\operatorname{dim}(Y)} \geq c^{-1} \operatorname{deg}(Y \cdot H) \cdot \delta^{\operatorname{dim}(Y \cdot H)},
\end{aligned}
$$

d'où résulte qu'une au moins des composantes isolées $Z^{\prime}$ de $Y \cdot H \subset Y$, de dimension $\operatorname{dim}(Y)-1 \geq d$, satisfait $\operatorname{Ord}_{\phi}\left(Z^{\prime}\right)>c^{-1} \operatorname{deg}\left(Z^{\prime}\right) \delta^{\operatorname{dim}\left(Z^{\prime}\right)}$. De plus, $H_{g}\left(Z^{\prime} ; \delta\right) \leq H_{g}(Y \cdot H ; \delta)<H_{g}(Y ; \delta) \quad$ (car $H$ est de degré $\delta$ et ne contient pas $Y$ ) et $\operatorname{deg}\left(Z^{\prime}\right) \leq \operatorname{deg}(Y) \cdot \delta=\operatorname{deg}(Y) \cdot \delta^{\operatorname{dim}(Y)-\operatorname{dim}\left(Z^{\prime}\right)}$.

- $\max _{\alpha \in Y(\bar{C})} \operatorname{Ord}_{z}(\alpha \wedge \phi) \leq c^{-1} \operatorname{deg}(Y) \cdot \delta^{\operatorname{dim}(Y)}:$ comme $Z \subset Y$ ceci entraîne $\max _{\alpha \in Z(\bar{C})} \operatorname{Ord}_{z}(\alpha \wedge \phi) \leq c^{-1} \operatorname{deg}(Y) \cdot \delta^{\operatorname{dim}(Y)}$. À nouveau les théorèmes de Bézout (voir [17], chap. 3, prop 4.11, avec $h(Z)=h(H)=\nu=0)$ montrent $\operatorname{deg}(Z \cdot H) \leq$ $\operatorname{deg}(Z) . \delta \leq \delta^{\operatorname{codim}(Z \cdot H)}$ et

$$
\operatorname{Ord}_{\phi}(Z \cdot H) \geq \operatorname{Ord}_{\phi}(Z)>c^{-1} \operatorname{deg}(Z) \cdot \delta^{\operatorname{dim}(Z)} \geq c^{-1} \operatorname{deg}(Z \cdot H) \cdot \delta^{\operatorname{dim}(Z \cdot H)},
$$

d'où résulte qu'une au moins des composantes isolées $Z^{\prime}$ de $Z \cdot H \subset Y$, de dimension $\operatorname{dim}(Z)-1 \geq d$, satisfait $\operatorname{Ord}_{\phi}\left(Z^{\prime}\right)>c^{-1} \operatorname{deg}\left(Z^{\prime}\right) \delta^{\operatorname{dim}\left(Z^{\prime}\right)}$. De plus, $H_{g}\left(Z^{\prime} ; \delta\right) \leq H_{g}(Z \cdot H ; \delta)<H_{g}(Z ; \delta)=H_{g}(Y ; \delta) \quad$ (car $H$ est de degré $\delta$ et ne contient pas $Z$ ) et $\operatorname{deg}\left(Z^{\prime}\right) \leq \operatorname{deg}(Z) \cdot \delta=\operatorname{deg}(Z) \cdot \delta^{\operatorname{dim}(Z)-\operatorname{dim}\left(Z^{\prime}\right)}$.

Si $H_{g}(Z ; \delta)<H_{g}(Y ; \delta)$ on pose $Z^{\prime}=Z$ et dans tous les cas on a montré l'existence de $Z^{\prime}$ de dimension $\geq d$ contenue dans $Y$, satisfaisant $H_{g}\left(Z^{\prime} ; \delta\right)<$ $H_{g}(Y ; \delta), \operatorname{deg}\left(Z^{\prime}\right) \leq \operatorname{deg}(Z) \cdot \delta^{\operatorname{dim}(Z)-\operatorname{dim}\left(Z^{\prime}\right)}$ et $\operatorname{Ord}_{\phi}\left(Z^{\prime}\right)>c^{-1} \operatorname{deg}\left(Z^{\prime}\right) \delta^{\operatorname{dim}\left(Z^{\prime}\right)}$.

Concluons cette section par une scolie.

Scolie 5 - Soit $V$ et $H$ un ensemble algébrique fermé et une hypersurface dans $\boldsymbol{P}_{n}$ respectivement, si $V \not \subset H$ toute composante isolée $Y$ de $V \cap H$ est composante isolée de $Y^{\prime} \cap H$ pour toutes les composantes $Y^{\prime}$ de $V$ contenant $Y$. 
Démonstration - En effet, pour toute composante $Y^{\prime}$ de $V$ les composantes de $Y^{\prime} \cap H$ sont contenues dans $V \cap H$ et donc dans au moins une composante isolée de $V \cap H$. Si pour une composante $Y^{\prime}$ de $V$ contenant $Y$ il existe une composante de $Y^{\prime} \cap H$ contenant strictement $Y$, alors $Y$ est strictement contenue dans une composante isolée de $V \cap H$ et ne peut être isolée dans cette dernière intersection. $\square$

\section{$\S 4$. Preuve du théorème 1 et de ses corollaires}

On veut maintenant montrer qu'il existe une variété $X$ de dimension $d \geq 1$ donnée, contenue dans $X_{0}$ et telle que $\operatorname{Ord}_{\phi}(X)>c_{n}^{-1} \operatorname{deg}(X) \delta^{d}$ et $\operatorname{deg}(X) \leq$ $\operatorname{deg}\left(X_{0}\right) \delta^{d_{0}-d}$, raisonnons par l'absurde et montrons la proposition suivante.

Proposition 6 - Soit $c \geq 1$ un réel, $\delta \in \boldsymbol{N}^{*}$ et $X_{0} \subset \boldsymbol{P}_{n}$ défini et de dimension $d_{0}$ sur $\boldsymbol{k}$, satisfaisant

i) $\operatorname{Ord}_{\phi}\left(X_{0}\right)>c^{-1} \operatorname{deg}\left(X_{0}\right) \delta^{d_{0}}$;

ii) pour toute sous-variété $Y \subset X_{0}$ incomplètement définie sur $\boldsymbol{k}$ dans $X_{0}$ par des formes de degré $\leq \delta$ on $a: H_{g}(Y ; \delta)>2 c^{-1} \operatorname{deg}(Y) \delta^{\operatorname{dim}(Y)}$.

Alors, pour tout entier $1 \leq d \leq d_{0}$,

- ou bien il existe $Z \subset X_{0}$ de dimension $d$ satisfaisant $\operatorname{deg}(Z) \leq \operatorname{deg}\left(X_{0}\right) \delta^{d_{0}-d}$ et $\operatorname{Ord}_{\phi}(Z)>c^{-1} \operatorname{deg}(Z) \delta^{d}$;

- ou bien il existe des hypersurfaces $H_{1}, \ldots, H_{d_{0}-d}$ de degré $\leq \delta$ telles que si on pose $V_{0}=X_{0}$ puis pour $i=1, \ldots, d_{0}-d, V_{i}=V_{0} \cap H_{1} \cap \ldots \cap H_{i}$ et $X_{i}$ l'union des composantes isolées de $V_{i}$ contenant une variété $Z^{\prime} \subset X_{0}$ de dimension $\geq d$ satisfaisant $\operatorname{deg}\left(Z^{\prime}\right) \leq \operatorname{deg}\left(X_{0}\right) \delta^{d_{0}-\operatorname{dim}\left(Z^{\prime}\right)}$ et $\operatorname{Ord}_{\phi}\left(Z^{\prime}\right)>c^{-1} \operatorname{deg}\left(Z^{\prime}\right) \delta^{\operatorname{dim}\left(Z^{\prime}\right)}$, on a $X_{i}$ non vide, pur de dimension $d_{0}-i$ et $\operatorname{deg}\left(X_{i}\right) \leq \operatorname{deg}\left(X_{0}\right) \delta^{i}$ pour $i=0, \ldots, d_{0}-d$.

Démonstration du théorème 1 - On applique la proposition 6 ci-dessus (avec $c=c_{n}$ ), l'hypothèse i) vient de celle du théorème 1 et l'hypothèse ii) résulte du lemme 3 (avec $c=c_{n}$ et $\left.\delta=\delta^{\prime} \geq 2(n+1)\right)$ car une variété incomplètement définie sur $k$ par des formes de degré $\leq \delta$ dans une variété elle-même définie par des formes de degré $\leq \delta$, est incomplètement définie dans $\boldsymbol{P}_{n}(\boldsymbol{k})$ par des formes de degré $\leq \delta$. Comme $X_{d_{0}-d}$ est non vide de dimension $d_{0}-\left(d_{0}-d\right)=d$ et degré $\leq \operatorname{deg}\left(X_{0}\right) \delta^{d_{0}-\operatorname{dim}(Z)}$, une variété $Z^{\prime}$ de dimension $\geq d$, contenue dans l'une quelconque de ses composantes est nécessairement de dimension $d$, ce qui montre que la première alternative de la conclusion de la proposition 6 résulte aussi de la seconde. Cette conclusion est celle du théorème 1 qui se trouve ainsi établi.

Démonstration de la proposition 6 - On démontre la proposition en supposant la première alternative de la conclusion non réalisée et en construisant les hypersurfaces $H_{i}$ par récurrence sur $i$, le pas $i=0$ étant banal avec $V_{0}=X_{0}$, grâce à l'hypothèse 
i). Supposons donc construites les hypersurfaces $H_{1}, \ldots, H_{i}$ pour $0 \leq i \leq d_{0}-d-1$. On commence par montrer qu'il existe une variété $Z^{\prime}$ de dimension $\geq d$, contenue dans $X_{i}$ et de degré $\leq \operatorname{deg}\left(X_{0}\right) \delta^{d_{0}-\operatorname{dim}\left(Z^{\prime}\right)}$, satisfaisant $H_{g}\left(Z^{\prime} ; \delta\right)<H_{g}\left(Y^{\prime} ; \delta\right)$ pour toute composante $Y^{\prime}$ de $X_{i}$.

Soit $Y$ une composante de $X_{i}$ réalisant le minimum de $H_{g}(Y ; \delta)$ sur toutes les composantes de $X_{i}$, considérons une variété $Z$ contenue dans $Y$ de dimension $\geq d$ maximale et de degré $\leq \operatorname{deg}\left(X_{0}\right) \delta^{d_{0}-\operatorname{dim}(Z)}$, satisfaisant $\operatorname{Ord}_{\phi}(Z)>$ $c^{-1} \operatorname{deg}(Z) \delta^{\operatorname{dim}(Z)}$. En fait $\operatorname{dim}(Z)>d$ d'après l'hypothèse faite en tête de démonstration et, comme $Y$ (composante isolée de $V_{i}$ ) est incomplètement définie dans $X_{0}$ par des formes de degré $\leq \delta$, on peut, grâce à l'hypothèse ii), appliquer la proposition 4 qui montre l'existence d'une variété $Z^{\prime}$ de dimension $\geq d$ et degré $\leq \operatorname{deg}(Z) \delta^{\operatorname{dim}(Z)-\operatorname{dim}\left(Z^{\prime}\right)} \leq \operatorname{deg}\left(X_{0}\right) \delta^{d_{0}-\operatorname{dim}\left(Z^{\prime}\right)}$, contenue dans $Y \subset X_{i}$, satisfaisant $\operatorname{Ord}_{\phi}\left(Z^{\prime}\right)>c^{-1} \operatorname{deg}\left(Z^{\prime}\right) \delta^{\operatorname{dim}\left(Z^{\prime}\right)}$ et $H_{g}\left(Z^{\prime} ; \delta\right)<H_{g}(Y ; \delta)=\min _{Y^{\prime}} H_{g}\left(Y^{\prime} ; \delta\right)$, où le minimum est pris sur toutes les composantes $Y^{\prime}$ de $X_{i}$.

Il existe donc (par un lemme d'évitement) une hypersurface $H_{i+1}$ de degré $\delta$ contenant $Z^{\prime}$ et aucune composante de $X_{i}$, ce qui entraîne immédiatement que $X_{i+1}$ n'est pas vide (au moins une composante isolée de $V_{i+1}$ doit contenir $Z^{\prime}$ ). De plus, toute composante isolée $Y$ de $V_{i+1}$ est composante isolée de $Y^{\prime} \cap H_{i+1}$ avec $Y^{\prime}$ composante isolée de $V_{i}$ (voir scolie 5). Mais, si $Y$ contient une variété de dimension $\geq d$ satisfaisant $\operatorname{deg}(Z) \leq \operatorname{deg}\left(X_{0}\right) \delta^{d_{0}-\operatorname{dim}(Z)}$ et $\operatorname{Ord}_{\phi}(Z)>c^{-1} \operatorname{deg}(Z) \delta^{\operatorname{dim}(Z)}$, il en est bien évidemment de même de $Y^{\prime}$ qui est donc composante de $X_{i}$. Ainsi, comme par hypothèse de récurrence on a $X_{i}$ pur de dimension $d_{0}-i$ et $\operatorname{deg}\left(X_{i}\right) \leq \operatorname{deg}\left(X_{0}\right) \delta^{i}$, il vient $\operatorname{dim}(Y)=\operatorname{dim}\left(Y^{\prime}\right)-1=d_{0}-i-1$, puis que $X_{i+1} \subset X_{i} \cap H_{i+1}$ est pur de dimension $d_{0}-i-1$ et, comme $H_{i+1}$ intersecte proprement $X_{i}$, on a enfin $\operatorname{deg}\left(X_{i+1}\right) \leq \operatorname{deg}\left(X_{i}\right) \cdot \delta \leq \operatorname{deg}\left(X_{0}\right) \delta^{i+1}$, ce qui achève la récurrence et démontre la proposition 6 .

Remarque - Dans la démonstration ci-dessus, lorsque $X_{0}=\boldsymbol{P}_{n}$, on peut choisir successivement les hypersurfaces $H_{i+1}$ de degré minimal $1 \leq \delta_{i+1} \leq \delta$, contenant une sous-variété $Z$ telle que $\operatorname{Ord}(Z)>c_{n}^{-1} \operatorname{deg}(Z) \delta^{\operatorname{dim}(Z)}$ et $\operatorname{deg}(Z) \leq \delta^{\operatorname{codim}(Z)}$, contenue dans $X_{i}$ et aucune composante de $X_{i}$. Ainsi $H_{i+1}$ ne contient aucune composante de $X_{i-1}$, car $X_{i} \subset X_{i-1}$, et on a $\delta_{i} \leq \delta_{i+1}$ (par la minimalité de $\delta_{i}$ ).

On montre alors par récurrence

$$
\operatorname{deg}\left(X_{i+1}\right) \geq \operatorname{deg}(Y) \geq\left(2^{n} c_{n}\right)^{-(i+1)} \delta_{1} \ldots \delta_{i+1} \geq\left(2^{n} c_{n}\right)^{-(i+1)} \operatorname{deg}\left(X_{i+1}\right)
$$

pour toute composante $Y$ de $X_{i+1}$. Les première et troisième inégalités sont claires car $\operatorname{deg}(Y) \leq \operatorname{deg}\left(X_{i+1}\right) \leq \delta_{i+1} \operatorname{deg}\left(X_{i}\right)$. Si $\delta_{i+1}=1$ alors $\delta_{1}=\ldots=\delta_{i}=1$, $Y=X_{i+1}=V_{i+1}$ est une variété linéaire et la seconde inégalité est également claire. Sinon $\delta_{i+1}>1$ et supposons cette seconde inégalité en défaut pour $i+1$ mais réalisée pour $0 \leq i<n-d$, on a pour toute composante $Y^{\prime}$ de $X_{i}$, par le lemme 3 ( $Y^{\prime}$ 
étant une composante isolée de $V_{i}$ est incomplètement définie sur $\boldsymbol{k}$ dans $\boldsymbol{P}_{n}$ par des formes de degré $\leq \delta_{i+1}$ ) et l'hypothèse de récurrence :

$$
\begin{array}{rlr}
H_{g}\left(Y ; \delta_{i+1}-1\right) & \leq \operatorname{deg}(Y) \delta_{i+1}^{n-i-1} & \text { (majoration du lemme } 3 \text { appliqué à } Y \text { ) } \\
& <\left(2^{n} c_{n}\right)^{-(i+1)} \delta_{1} \ldots \delta_{i} \delta_{i+1}^{n-i} & \text { (négation de la seconde inégalité de }(1)) \\
& <\left(2^{n} c_{n}\right)^{-1} \operatorname{deg}\left(Y^{\prime}\right) \delta_{i+1}^{n-i} & \text { (seconde inégalité de } \left.(1) \text { pour } Y^{\prime}\right) \\
& <H_{g}\left(Y^{\prime} ; \delta_{i+1}-1\right) & \text { (minoration du lemme } \left.3 \text { appliqué à } Y^{\prime}\right),
\end{array}
$$

en contradiction avec le choix minimal de $\delta_{i+1}$. Ceci entraîne que, lorsque $X_{0}=\boldsymbol{P}_{n}$, le nombre de composantes de $X_{i}$ est $\leq\left(2^{n} c_{n}\right)^{i}$.

Passons aux preuves des corollaires.

Démonstration $d u$ corollaire 2 - On applique le théorème 1 avec $d=1$, soit $Y=$ $X_{d_{0}-1} \subset X_{0}$ la variété de dimension 1 et degré $\leq \operatorname{deg}\left(X_{0}\right) \delta^{d_{0}-1}$, satisfaisant $\operatorname{Ord}_{\phi}(Y)>c^{-1} \operatorname{deg}(Y) \delta$, fournie par le théorème 1. Comme $Y$ est définie sur $\boldsymbol{k}$ elle possède des points définis sur $\boldsymbol{k}$ et ne peut donc être contenue dans l'hyperplan d'équation $\phi_{0} X_{1}=\phi_{1} X_{0}$, car $\frac{\phi_{1}}{\phi_{0}} \notin \boldsymbol{k}$. L'intersection $Z:=Y \cap \mathcal{Z}\left(\phi_{0} x_{1}-\phi_{1} x_{0}\right)$ est définie et de dimension 0 sur $\boldsymbol{k}(z)$. Il résulte des théorèmes de Bézout (voir [17], chap. 3, prop 4.11, avec $h(Y)=\nu=0, \operatorname{Ord}_{\phi}(H)=\infty$ et $\left.h(H)=h\left(\phi_{0}: \phi_{1}\right)\right)$ qu'on a $\operatorname{deg}(Z) \leq \operatorname{deg}(Y), h(Z) \leq h\left(\phi_{0}: \phi_{1}\right) \operatorname{deg}(Y)$ et

$$
\operatorname{Ord}_{\phi}(Z) \geq \operatorname{Ord}_{\phi}(Y) \geq c_{n}^{-1} \operatorname{deg}(Y) \delta \geq\left(2 c_{n}\right)^{-1}\left(\frac{h(Z)}{h\left(\phi_{0}: \phi_{1}\right)}+\operatorname{deg}(Z)\right) \delta
$$

Démonstration du lemme de transfert-On pose $X_{0}=\mathcal{Z}(P)$ et $^{\dagger}$

$$
\delta:=\left[b^{\frac{1}{n-1}} d^{\circ} P^{\frac{\mu-1}{n-1}}\right] \geq d^{\circ} P
$$

de sorte que $\delta \geq 2(n+1)$,

$$
\operatorname{Ord}_{\phi}(\mathcal{Z}(P))=\operatorname{ord}_{z}(P \circ \phi)-d^{\circ} P . \operatorname{ord}_{z}(\phi)>b d^{\circ} P^{\mu} \geq c_{n}^{-1} d^{\circ} P \delta^{n-1}
$$

et $X_{0}$ est une variété de dimension $d_{0}=n-1$ telle que $\operatorname{Ord}_{\phi}\left(X_{0}\right)>c_{n}^{-1} \operatorname{deg}\left(X_{0}\right) \delta^{n-1}$. On déduit du corollaire 2 qu'il existe un cycle $Z \subset \mathcal{Z}(P)(\overline{\boldsymbol{k}(z)})$ de dimension 0 et défini sur $\boldsymbol{k}(z)$, tel que

$$
\begin{aligned}
\operatorname{deg}(Z) & \leq \operatorname{deg}\left(X_{0}\right) \delta^{n-2} \leq b^{\frac{n-2}{n-1}} d^{\circ} P^{1+\frac{(\mu-1)(n-2)}{n-1}}=b^{\frac{n-2}{n-1}} d^{\circ} P^{\frac{1+\mu(n-2)}{n-1}} \\
h(Z) & \leq h\left(\phi_{0}: \phi_{1}\right) \operatorname{deg}\left(X_{0}\right) \delta^{n-2} \leq h\left(\phi_{0}: \phi_{1}\right) b^{\frac{n-2}{n-1}} d^{\circ} P^{\frac{1+\mu(n-2)}{n-1}}
\end{aligned}
$$

$\dagger$ Les crochets $[\star]$ désignent la partie entière d'un élément $\star$ de $\boldsymbol{R}$, c'est-à-dire le plus grand entier relatif inférieur ou égal à $\star$. 
et

$$
\sum_{\alpha \in Z} \operatorname{Ord}_{z}(\alpha \wedge \phi)>\frac{1}{2 c_{n}}\left(\frac{h(Z)}{h\left(\phi_{0}: \phi_{1}\right)}+\operatorname{deg}(Z)\right) \delta .
$$

On vérifie en particulier $d^{\circ} P \geq\left(\frac{1}{2}\left(\frac{h(Z)}{h\left(\phi_{0}: \phi_{1}\right)}+\operatorname{deg}(Z)\right)\right)^{\frac{n-1}{1+\mu(n-2)}} b^{-\frac{n-2}{1+\mu(n-2)}}$ et on conclut

$$
\begin{aligned}
\sum_{\alpha \in Z} \operatorname{Ord}_{z}(\alpha \wedge \phi) & >\frac{1}{2 c_{n}}\left(\frac{h(Z)}{h\left(\phi_{0}: \phi_{1}\right)}+\operatorname{deg}(Z)\right) \delta \\
& >\frac{1}{4 c_{n}}\left(\frac{h(Z)}{h\left(\phi_{0}: \phi_{1}\right)}+\operatorname{deg}(Z)\right) b^{\frac{1}{n-1}} d^{\circ} P^{\frac{\mu-1}{n-1}} \\
& >\frac{b^{\frac{1}{n-1}-\frac{(\mu-1)(n-2)}{(n-1)(1+\mu(n-2))}}}{2 c_{n}}\left(\frac{1}{2}\left(\frac{h(Z)}{h\left(\phi_{0}: \phi_{1}\right)}+\operatorname{deg}(Z)\right)\right)^{1+\frac{\mu-1}{1+\mu(n-2)}}
\end{aligned}
$$

\section{$\S 5$. Régularité et plongements mixtes}

Afin de généraliser les résultats des paragraphes antérieurs aux sous-variétés des espaces multiprojectifs nous devons établir une version multihomogène du lemme 3 . C'est ce que nous faisons ici en nous ramenant au cas homogène par des plongements mixtes. Posons les notations que nous utiliserons pour ce contexte dans ce paragraphe et le suivant.

Soit $n_{1}, \ldots, n_{q} \in \boldsymbol{N}^{*}$, on considère l'espace multiprojectif $\boldsymbol{P}:=\boldsymbol{P}_{n_{1}} \times \ldots \times \boldsymbol{P}_{n_{q}}$ de dimension $n:=n_{1}+\ldots+n_{q}$ et on note $A:=\boldsymbol{k}\left[\boldsymbol{x}_{1}, \ldots, \boldsymbol{x}_{q}\right]$ son anneau de coordonnées sur $\boldsymbol{k}$, avec $\boldsymbol{x}_{i}:=\left(x_{i, 0}, \ldots, x_{i, n_{i}}\right)$ pour $i=1, \ldots, q$. Soit $\delta_{1}, \ldots, \delta_{q} \in$ $\boldsymbol{N}^{*}$ et $N+1:=\prod_{i=1}^{q}\left(\begin{array}{c}\delta_{i}+n_{i} \\ n_{i}\end{array}\right)$, on considère le plongement mixte

$$
\begin{aligned}
& \begin{array}{llll}
\sigma: & \boldsymbol{P} & \longrightarrow & \boldsymbol{P}_{N}
\end{array} \\
& \left(\boldsymbol{x}_{1}, \ldots, \boldsymbol{x}_{q}\right) \longmapsto\left(\ldots: \prod_{i=1}^{q} \prod_{j=1}^{n_{i}} x_{i, j}^{\alpha_{i, j}}: \ldots\right)_{\substack{\alpha_{i} \in N^{n_{i}} \\
\left|\alpha_{i}\right|=\delta_{i}, i=0, \ldots, q}}
\end{aligned}
$$

composé des plongements de Veronese

$$
\begin{array}{clc}
v_{i}: \quad \boldsymbol{P}_{n_{i}} & \longrightarrow & \boldsymbol{P}_{\left(\begin{array}{c}
\delta_{i}+n_{i} \\
n_{i}
\end{array}\right)} \\
\left(x_{i, 0}, \ldots, x_{i, n_{i}}\right) & \longmapsto & \left(\ldots: \prod_{j=0}^{n_{i}} x_{i, j}^{\alpha_{j}}: \ldots\right)_{\substack{\alpha \in N^{n_{i}} \\
|\alpha|=\delta_{i}}}^{n_{i, j}},
\end{array},
$$

pour $i=1, \ldots, q$, et du plongement de Segre

$$
\begin{aligned}
\boldsymbol{P}_{\left(\begin{array}{c}
\delta_{1}+n_{1} \\
n_{1}
\end{array}\right)} \times \ldots \times \boldsymbol{P}_{\left(\begin{array}{c}
\delta_{q}+n_{q} \\
n_{q}
\end{array}\right)} & \longrightarrow \\
\left(\boldsymbol{y}_{1}, \ldots, \boldsymbol{y}_{q}\right) & \longmapsto\left(\ldots: \prod_{i=1}^{q} y_{i, \alpha_{i}}: \ldots\right)
\end{aligned}
$$

Commençons par le calcul suivant de la régularité de $\sigma(\boldsymbol{P})$, qui m'a été fourni par M. Chardin. 
Proposition 7 - Soit $\sigma$ le plongement mixte décrit ci-dessus, alors la régularité de Castelnuovo-Mumford de $\sigma(\boldsymbol{P}) \subset \boldsymbol{P}_{N}$ est égale $\grave{a}^{\dagger}$

$$
n+1+\min _{i=1, \ldots, q}\left(\left[-\frac{n_{i}+1}{\delta_{i}}\right]\right) \leq n
$$

Démonstration - Pour $i=1, \ldots, q$ notons $A_{i}=\boldsymbol{k}\left[x_{i, 0}, \ldots, x_{i, n_{i}}\right]$ l'anneau des coordonnées de $\boldsymbol{P}_{n_{i}}$ et $A_{i}^{\left(\delta_{i}\right)}$ celui de $v_{i}\left(\boldsymbol{P}_{n_{i}}\right)$. Ainsi $A_{i}^{\left(\delta_{i}\right)}$ est la somme directe des parties de degrés divisibles par $\delta_{i}$ de $A_{i}$ tandis que l'anneau $B$ de $\sigma(\boldsymbol{P})$ est la somme directe des produits tensoriels des parties graduées de même degré de $A_{1}^{\left(\delta_{1}\right)}, \ldots, A_{q}^{\left(\delta_{q}\right)}$, notée $B=A_{1}^{\left(\delta_{1}\right)_{\sharp}} \ldots \sharp A_{q}^{\left(\delta_{q}\right)}$. Au niveau des parties graduées on a $B_{\nu}=\left(A_{1}\right)_{\delta_{1} \nu} \otimes \ldots \otimes\left(A_{q}\right)_{\delta_{q} \nu}$.

La régularité de Castelnuovo-Mumford d'un module gradué est définie comme le plus grand des nombres égaux aux plus petits degrés en lesquels les parties homogènes de chaque groupe de cohomologie locale du module sont nulles, augmenté de l'indice de ce groupe de cohomologie moins 1, i.e. la régularité du $A$-module $M$ est $\max _{h}\left(h-1+\min \left(\nu ; H_{\mathfrak{m}}^{h}(M)_{\geq \nu}=0\right)\right)$. Pour calculer la régularité de $X$ nous devons donc écrire les groupes de cohomologie locale de l'anneau $B$.

Chaque anneau $A_{i}$ étant Cohen-Macaulay ses groupes de cohomologie locale d'indice distinct de $n_{i}+1$ sont nuls, il en est donc de même pour $A_{i}^{\left(\delta_{i}\right)}$. Comme $n_{i} \geq 1$ on a en particulier $H_{\mathfrak{m}_{i}}^{0}\left(A_{i}^{\left(\delta_{i}\right)}\right) \simeq H_{\mathfrak{m}_{i}}^{1}\left(A_{i}^{\left(\delta_{i}\right)}\right) \simeq 0$, en itérant le théorème 4.1 .5 de [8] par récurrence sur $q$ on vérifie $H_{\mathfrak{m}}^{0}(B) \simeq H_{\mathfrak{m}}^{1}(B) \simeq 0$ et pour tout $h \geq 2$ :

$$
H_{\mathfrak{m}}^{h}(B)=\bigoplus_{\substack{h_{1}, \ldots, h_{q} \in N^{*} \\
h_{1}+\ldots+h_{q}=h+q-1}} M_{1}\left(h_{1}\right) \sharp \ldots \sharp M_{q}\left(h_{q}\right) \quad \text { où } \quad M_{i}\left(h_{i}\right)=\left\{\begin{array}{ll}
A_{i}^{\left(\delta_{i}\right)} & \text { si } h_{i}=1 \\
H_{\mathfrak{m}_{i}}^{h_{i}}\left(A_{i}^{\left(\delta_{i}\right)}\right) & \text { si } h_{i}>1
\end{array} .\right.
$$

En considérant les parties graduées de degré $\nu$ on a

$$
H_{\mathfrak{m}}^{h}(B)_{\nu}=\bigoplus_{\substack{h_{1}, \ldots, h_{q} \in N^{*} \\ h_{1}+\ldots+h_{q}=h+q-1}} M_{1}\left(h_{1}\right)_{\nu} \otimes \ldots \otimes M_{q}\left(h_{q}\right)_{\nu}
$$

Les modules $M_{i}(k)$ sont nuls pour $k \neq 1, n_{i}+1$ et $M_{i}\left(n_{i}+1\right)_{\nu}=H_{\mathfrak{m}_{i}}^{n_{i}+1}\left(A_{i}\right)_{\delta_{i} \nu}=0$ dès que $\delta_{i} \nu>-n_{i}-1$ (la régularité de $A_{i}$ est 0 ) tandis que $M_{i}(1)_{\nu}=\left(A_{i}\right)_{\delta_{i} \nu}=$ 0 lorsque $\nu<0$. Ceci entraîne $H_{\mathfrak{m}}^{h}(B)=0$ pour tout $h \neq n+1$ et, de plus, $H_{\mathfrak{m}}^{n+1}(B)_{\nu}=0$ pour $\nu>\min _{i=1, \ldots, q}\left(\left[-\frac{n_{i}+1}{\delta_{i}}\right]\right)$. On vérifie également que pour $\nu=\min _{i=1, \ldots, q}\left(\left[-\frac{n_{i}+1}{\delta_{i}}\right]\right)$ on a $H_{\mathfrak{m}}^{n+1}(B)_{\nu}=\bigotimes_{i=1}^{q} H_{\mathfrak{m}_{i}}^{n_{i}+1}\left(A_{i}\right)_{\delta_{i} \nu} \neq 0$ car $H_{\mathfrak{m}_{i}}^{n_{i}+1}\left(A_{i}\right)_{\nu^{\prime}} \neq 0$ pour tout $\nu^{\prime} \leq-n_{i}-1$ et $i=1, \ldots, q$.

$\dagger$ Les crochets $[\star]$ désignent la partie entière d'un élément $\star$ de $\boldsymbol{R}$, c'est-à-dire le plus grand entier relatif inférieur ou égal à $\star$. 
En revenant à la définition de la régularité indiquée plus haut on obtient le résultat annoncé.

Remarque - Lorsque $\delta_{i}>n_{i}$ pour $i=1, \ldots, q$ la régularité de $\sigma(\boldsymbol{P})$ est égale à $n$, tandis que pour $\delta_{1}=\ldots=\delta_{q}=1$ elle est égale à $n-\max \left(n_{1} ; \ldots ; n_{q}\right)$. Dans les autres cas elle prend une valeur intermédiaire.

Soit $X$ une sous-variété projective de $\boldsymbol{P}(\boldsymbol{k})$ et $\delta=\left(\delta_{1}, \ldots, \delta_{q}\right) \in\left(\boldsymbol{N}^{*}\right)^{q}$, nous dirons qu'une sous-variété projective $Z$ de $X$ définie sur $\boldsymbol{k}$ est incomplètement définie sur $\boldsymbol{k}$ dans $X$ par des formes de multidegrés $\leq \delta$ si $Z$ est composante isolée du cycle intersection de $X$ et d'hypersurfaces de degrés $\leq \delta$ (définies sur $\boldsymbol{k}$ ).

On établit maintenant les estimations de fonctions de Hilbert utiles.

Théorème $\mathbf{8}$ - Soit $X \subset \boldsymbol{P}$ une variété incomplètement définie sur $\boldsymbol{k}$ dans $\boldsymbol{P}$ par des équations de multidegré $\leq\left(\delta_{1}, \ldots, \delta_{q}\right)$, alors

$$
\begin{gathered}
H_{g}(\sigma(X) ; n+1) \geq \operatorname{deg}(\sigma(X))(\operatorname{dim}(X)+1) \\
H_{g}(\sigma(X) ; 1) \leq \operatorname{deg}(\sigma(X))+\operatorname{dim}(X) .
\end{gathered}
$$

Démonstration - Rappelons les notions de $(m, \mathfrak{b})$-régularité et $(m, \mathfrak{b})$-perfection introduites dans [4], définition 1. La variété $\sigma(X)$ est définie dans $\sigma(\boldsymbol{P})$ par des équations linéaires et, comme $\sigma(\boldsymbol{P})$ est $(n,(1))$-parfait d'après la proposition 7 , il résulte de la proposition 1 de [4] que $\sigma(X)$ est composante d'un schéma équidimensionnel (découpé dans $\sigma(\boldsymbol{P})$ par $n-\operatorname{dim}(X)$ formes linéaires s'annulant sur $\sigma(X)$, assez générales) dont l'anneau est $(n, \mathfrak{b})$-parfait (pour un certain idéal $\mathfrak{b} \subset \boldsymbol{k}\left[x_{0}, \ldots, x_{N}\right]$ ). On applique alors la proposition 4 de loc. cit. en degré $n+1$, qui donne

$$
H_{g}(\sigma(X) ; n+1) \geq \operatorname{deg}(\sigma(X))\left(\begin{array}{c}
\operatorname{dim}(X)+1 \\
\operatorname{dim}(X)
\end{array}\right)
$$

Pour la seconde inégalité, un théorème de géométrie classique, voir [17], chap. 9, lem. 1.1, montre directement le résultat.

Si $X$ est une sous-variété projective de $\boldsymbol{P}(\boldsymbol{k})$ (i.e. un ensemble algébrique réduit et irréductible sur $\boldsymbol{k}$ ), de dimension $d$, on note pour $\delta=\left(\delta_{1}, \ldots, \delta_{q}\right) \in \boldsymbol{N}^{q}$

$$
\operatorname{deg}(X ; \delta):=\sum_{\substack{\alpha \in N^{q} \\
|\alpha|=d}} d_{\alpha}(X) \cdot\left(\begin{array}{l}
d \\
\alpha
\end{array}\right) \cdot \delta_{1}^{\alpha_{1}} \ldots \delta_{q}^{\alpha_{q}}
$$

sa forme multidegré, où $d_{\alpha}(X)$ désigne le multidegré d'indice $\alpha$ de $X$. On a $d_{\alpha}(X)=0$ dès que $\alpha_{i}>\operatorname{dim}\left(\pi_{i}(X)\right)$ pour $1 \leq i \leq q$, avec $\pi_{i}$ la projection de $\boldsymbol{P}$ 
sur son $i$-ème facteur $\boldsymbol{P}_{n_{i}}$. Si $X$ est de dimension 1 , pour $\varepsilon_{i}:=(0, \ldots, 0,1,0, \ldots, 0)$ (le 1 étant en $i$-ème position), $d_{\varepsilon_{i}}(X)$ est le produit du degré de la projection de $X$ sur le $i$-ème facteur par le degré de la fibre générique de cette projection. Si $X=\mathcal{Z}(P)$ est une hypersurface d'équation $P \in A$, on a

$$
\operatorname{deg}(X ; \delta)=\sum_{i=1}^{q} d_{\boldsymbol{x}_{i}}^{\circ} P \cdot\left(\begin{array}{c}
n-1 \\
n_{1} \ldots n_{i}-1 \ldots n_{q}
\end{array}\right) \cdot \delta_{1}^{n_{1}} \ldots \delta_{i}^{n_{i}-1} \ldots \delta_{q}^{n_{q}}
$$

On notera encore $H_{g}(X ; \delta)$ la valeur en $\delta=\left(\delta_{1}, \ldots, \delta_{q}\right) \in \boldsymbol{N}^{q}$ de la fonction de Hilbert (géométrique) de $X$, i.e. la dimension sur $\boldsymbol{k}$ de l'espace des formes à coefficients dans $\boldsymbol{k}$, multihomogènes de multidegré $\delta$ par rapport aux groupes de variables $\left(\boldsymbol{x}_{1}, \ldots, \boldsymbol{x}_{q}\right)$.

Corollaire 9 - Soit $X \subset \boldsymbol{P}$ une variété de dimension $d$, définie sur $\boldsymbol{k}$. Pour $\delta \in \boldsymbol{N}^{q}$ on note $0=\varepsilon_{0}<\varepsilon_{1} \leq \ldots \leq \varepsilon_{n-d}<\varepsilon_{n-d+1}=\infty$ la suite des réels minimaux telle que $X$ soit génériquement intersection complète d'hypersurfaces de $\boldsymbol{P}$ définies sur $\boldsymbol{k}$ par des formes de multidegré $\varepsilon_{1} \delta, \ldots, \varepsilon_{n-d} \delta$ respectivement. Alors, si toutes les coordonnées de $\delta$ sont $\geq 2(n+1)^{n-d+2}$ on $a$

$$
H_{g}(X ; \delta) \geq(d+1)\left(2(n+1)^{n-d+2}\right)^{-n} \cdot \prod_{j=1}^{n-d} \max \left(\frac{1}{n+1} ; \varepsilon_{j}\right)^{-1} \cdot \operatorname{deg}(X ; \delta)
$$

En particulier, si $X$ est incomplètement définie sur $\boldsymbol{k}$ dans $\boldsymbol{P}$ par des formes de multidegré $\leq \delta$ on a

$$
(d+1) 2^{-n}(n+1)^{-n(n-d+2)} \cdot \operatorname{deg}(X ; \delta) \leq H_{g}(X ; \delta) \leq \operatorname{deg}(X ; \delta)+d .
$$

Démonstration - Extrayons de $\{0, \ldots, n-d+1\}$ le plus petit indice, noté $j_{0}$, tel que $(n+1) \varepsilon_{j_{0}}>1$ et le plus grand indice, noté $i_{0}$, tel que $(n+1)^{n-d+2} \varepsilon_{i_{0}} \leq 1$. On vérifie qu'il existe $i \in\left\{i_{0}, \ldots, j_{0}-1\right\}$ tel que $\varepsilon_{i+1}>(n+1) \varepsilon_{i}$. En effet, sinon on écrirait (en remarquant $j_{0}-i_{0} \leq n-d+1$ )

$$
\varepsilon_{j_{0}} \leq(n+1) \varepsilon_{j_{0}-1} \leq \ldots \leq(n+1)^{j_{0}-i_{0}} \varepsilon_{i_{0}} \leq \frac{1}{n+1}
$$

en contradiction avec le choix de $j_{0}$. On fixe $i$ le plus grand indice dans $\left\{i_{0}, \ldots, j_{0}-1\right\}$ tel que $\varepsilon_{i+1}>(n+1) \varepsilon_{i}$. On vérifie alors

$$
\frac{1}{n+1}<\varepsilon_{j_{0}} \leq(n+1) \varepsilon_{j_{0}-1} \leq \ldots \leq(n+1)^{j_{0}-i-1} \varepsilon_{i+1}
$$

d'où $\varepsilon_{i+1}>(n+1)^{i-j_{0}} \geq(n+1)^{-n+d-1}$. 
Comme $i<j_{0}$ on a $(n+1) \varepsilon_{i} \delta \leq \delta$ et $(n+1) \varepsilon_{i} \delta<\varepsilon_{i+1} \delta$, on peut choisir $\delta^{\prime} \in \boldsymbol{N}^{q}$ de longueur maximale satisfaisant $(n+1) \delta^{\prime} \leq \delta,(n+1) \delta^{\prime}<\varepsilon_{i+1} \delta$ et $\delta^{\prime} \geq \varepsilon_{i} \delta \quad$ (on notera que $\varepsilon_{i} \delta \in \boldsymbol{N}^{q}$ ). On vérifie

$$
\delta^{\prime} \geq(2(n+1))^{-1} \min \left(1 ; \varepsilon_{i+1}\right) \delta \geq \frac{1}{2}(n+1)^{i-j_{0}-1} \delta
$$

car les coordonnées de $(n+1)^{-1} \min \left(1 ; \varepsilon_{i+1}\right) \delta$ sont toutes $\geq 2$.

On considère l'union $Y$ des composantes, contenant $X$, du schéma défini par l'idéal engendré par toutes les formes de multidegré $<\varepsilon_{i+1} \delta$ qui s'annulent sur $X$. Comme $\varepsilon_{i}<\varepsilon_{i+1}$ et qu'il n'existe pas d'intersection complète au point générique de $X$ de formes de multidegré $<\varepsilon_{i+1} \delta$ de dimension $<n-i$ contenant $X$, on en déduit que $\operatorname{dim}(Y)=n-i$ et que $Y$ est incomplètement définie sur $\boldsymbol{k}$ dans $\boldsymbol{P}$ par des formes de multidegré $\leq \varepsilon_{i} \delta \leq \delta^{\prime}$. On peut donc appliquer le théorème 8 à $Y$ avec le multidegré $\delta^{\prime}$ et on vérifie (en notant que $H_{g}(\sigma(Y) ; n+1)=H_{g}\left(Y ;(n+1) \delta^{\prime}\right.$ ) et $\left.\operatorname{deg}(\sigma(Y))=\operatorname{deg}\left(Y ; \delta^{\prime}\right)\right)$

$$
H_{g}(X ; \delta) \geq H_{g}\left(X ;(n+1) \delta^{\prime}\right)=H_{g}\left(Y ;(n+1) \delta^{\prime}\right) \geq(d+1) \operatorname{deg}\left(Y ; \delta^{\prime}\right)
$$

car $(n+1) \delta^{\prime} \leq \delta$ et $(n+1) \delta^{\prime}<\varepsilon_{i+1} \delta$ pour la première inégalité et l'égalité respectivement. Mais, $X$ est une composante d'une intersection de $Y$ par des hypersurfaces de multidegré $\varepsilon_{i+1} \delta, \ldots, \varepsilon_{n-d} \delta$ et on a donc, d'après le théorème de Bézout multihomogène et avec la minoration (2) de $\delta^{\prime}$ (soit $\delta \leq 2(n+1)^{j_{0}-i+1} \delta^{\prime}$ ),

$$
\begin{aligned}
\operatorname{deg}(X ; \delta) & \leq \varepsilon_{i+1} \cdots \varepsilon_{n-d} \cdot \operatorname{deg}(Y ; \delta) \\
& \leq 2^{n-i}(n+1)^{(n-i)\left(j_{0}-i+1\right)} \cdot \prod_{j=i+1}^{n-d} \varepsilon_{j} \cdot \operatorname{deg}\left(Y ; \delta^{\prime}\right) \\
& \leq\left(2(n+1)^{n-d+2}\right)^{n} \cdot \prod_{j=1}^{n-d} \max \left(\frac{1}{n+1} ; \varepsilon_{j}\right) \cdot \operatorname{deg}\left(Y ; \delta^{\prime}\right) .
\end{aligned}
$$

Le premier résultat suit en combinant cette majoration avec (3). Pour la minoration du second il suffit de remarquer que si $X$ est incomplètement définie dans $\boldsymbol{P}$ par des formes de degré $\leq \delta$ alors tous les $\varepsilon_{j}$ sont $\leq 1$ et la majoration résulte directement du théorème 8 .

\section{$\S 6$. Le cas multiprojectif}

On reprend notre corps $\boldsymbol{k}$, commutatif et algébriquement clos, $z$ une variable sur $\boldsymbol{k}, C:=\boldsymbol{k}((z))$ et on note $\bar{C}$ la clôture algébrique de $C$. On étend les notations $\mathrm{du} \S 2 \mathrm{au}$ cas des produits d'espaces projectifs. 
Soit $n_{1}, \ldots, n_{q} \in \boldsymbol{N}^{*}$, on reprend l'espace multiprojectif $\boldsymbol{P}:=\boldsymbol{P}_{n_{1}} \times \ldots \times \boldsymbol{P}_{n_{q}}$ de dimension $n=n_{1}+\ldots+n_{q}$ et on note $A=\boldsymbol{k}\left[\boldsymbol{x}_{1}, \ldots, \boldsymbol{x}_{q}\right]$ son anneau de coordonnées sur $\boldsymbol{k}$, avec $\boldsymbol{x}_{i}=\left(x_{i, 0}, \ldots, x_{i, n_{i}}\right)$ pour $i=1, \ldots, q$.

Soit $X \subset \boldsymbol{P}$ une variété définie sur $\boldsymbol{k}$ de dimension $d, \delta=\left(\delta_{1}, \ldots, \delta_{q}\right) \in\left(\boldsymbol{N}^{*}\right)^{q}$ et $f_{X} \in \boldsymbol{k}\left[\boldsymbol{u}^{(1)}, \ldots, \boldsymbol{u}^{(d+1)}\right]$ une forme éliminante d'indice $(\delta, \ldots, \delta) \in\left(\left(\boldsymbol{N}^{*}\right)^{q}\right)^{d+1}$ de $X$ (voir [17], chap. 5), c'est une forme multihomogène symétrique en les variables $\boldsymbol{u}^{(j)}=\left(u_{i, k}^{(j)}\right)_{\substack{i=1, \ldots, q \\ k=0, \ldots, n_{i}}}$ et on a, avec les notations introduites au paragraphe précédent avant le corollaire $9, \operatorname{deg}(X ; \delta)=d^{\circ} f_{X} /(d+1) \in \mathbf{N}^{*}$.

Soit $\phi_{i}:=\left(\phi_{i, 0}, \ldots, \phi_{i, n_{i}}\right) \in \boldsymbol{P}_{n_{i}}(C)$ et $\Phi:=\left(\phi_{1}, \ldots, \phi_{q}\right) \in \boldsymbol{P}(C)$. On pose

$$
\operatorname{Ord}_{\Phi}(X):=\operatorname{ord}_{z}\left(\mathfrak{d}_{\Phi} f_{X}\right)-(d+1) \sum_{i=1}^{q} \delta_{i} \operatorname{ord}_{0}\left(\phi_{i}\right) \cdot \operatorname{deg}(X ; \delta)
$$

où $\quad \mathfrak{d}_{\Phi}: A\left[\boldsymbol{u}^{(1)}, \ldots, \boldsymbol{u}^{(d+1)}\right] \rightarrow C[\boldsymbol{s}]:=A\left[\boldsymbol{s}^{(1)}, \ldots, \boldsymbol{s}^{(d+1)}\right] \otimes_{\boldsymbol{k}} C$ est l'opérateur défini par $\mathfrak{d}_{\Phi}\left(u_{i, k}^{(j)}\right)=\sum_{\ell=0}^{n_{i}} s_{i, k, \ell}^{(j)} \phi_{i, \ell} \quad\left(\right.$ avec $s_{i, k, \ell}^{(j)}+s_{i, \ell, k}^{(j)}=0$ pour tous $i, j, k, \ell$, voir [17], chap. 5). On vérifie que cette définition ne dépend pas du choix de l'indice $\delta$ fixé (car aucune composante de $\delta$ n'est nulle), en reprenant la démonstration de la proposition $3 \mathrm{du}$ chapitre 7 de [17]. En particulier, si $X$ est une courbe dans

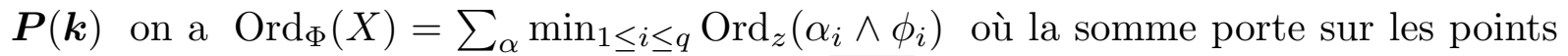
$\alpha=\left(\alpha_{1}, \ldots, \alpha_{q}\right) \in X \cap \mathcal{Z}\left(\mathfrak{d}_{\Phi} U_{1}\right)$ définis sur $\overline{C(\boldsymbol{s})}$. Tandis que si $X=\mathcal{Z}(P)$ est une hypersurface alors $\operatorname{Ord}_{\Phi}(X)=\operatorname{ord}_{z}(P(\Phi))-\sum_{i=1}^{q} d_{\boldsymbol{x}_{i}}^{\circ} P \cdot \operatorname{ord}_{z}\left(\phi_{i}\right)$.

Si $\alpha \in \boldsymbol{P}(\overline{\boldsymbol{k}(z)})$ et $\alpha_{1}, \ldots, \alpha_{D}$ sont ses conjugués sur $\boldsymbol{k}(z)$ on définit la forme multihauteur du cycle $Z:=\left\{\alpha_{1}, \ldots, \alpha_{D}\right\}$ par la formule $h(Z ; \delta):=$ $-\sum_{u \in \boldsymbol{P}_{1}(\overline{\boldsymbol{k}})} \operatorname{ord}_{z_{u}}\left(f_{Z}\right)$ où $f_{Z}$ désigne une forme éliminante d'indice $\delta \in\left(\boldsymbol{N}^{*}\right)^{q}$ de $Z$ et $z_{u}=z-v$ si $u=(1: v)$ et $z_{u}=1 / z$ si $u=(0: 1)$. On a encore $h(Z ; \delta)=\sum_{i=1}^{q} \delta_{i} h\left(\pi_{i}(Z)\right)$ où $\pi_{i}: \boldsymbol{P} \rightarrow \boldsymbol{P}_{n_{i}}$ désigne la projection sur le $i$-ème facteur de $\boldsymbol{P}$ et $\operatorname{Ord}_{\Phi}(Z)=\sum_{\alpha \in Z} \min _{1 \leq i \leq q}\left(\operatorname{Ord}_{z}\left(\pi_{i}(\alpha) \wedge \pi_{i}(\phi)\right)\right)$.

Théorème 10 - Avec les notations ci-dessus, on note $c_{\boldsymbol{P}}:=2^{n+1}(n+1)^{n(n+2)}$. On se donne une variété $X_{0}$ de dimension $d_{0}$, définie sur $\boldsymbol{k}$ dans $\boldsymbol{P}$ par des formes de multidegrés $\leq \delta$ où $\min \left(\delta_{1}, \ldots, \delta_{q}\right) \geq 2(n+1)^{n+1}$ et satisfaisant

$$
\operatorname{Ord}_{\Phi}\left(X_{0}\right)>c_{\boldsymbol{P}}^{-1} \operatorname{deg}\left(X_{0} ; \delta\right)
$$

Alors, pour $i=0, \ldots, d_{0}-1$ il existe une variété $X_{i} \subset X_{0}$ de dimension $d_{0}-i$ et définie sur $\boldsymbol{k}$, satisfaisant $\operatorname{deg}\left(X_{i} ; \delta\right) \leq \operatorname{deg}\left(X_{0} ; \delta\right) \leq \delta_{1}^{n_{1}} \ldots \delta_{q}^{n_{q}}$ et

$$
\operatorname{Ord}_{\Phi}\left(X_{i}\right)>c_{\boldsymbol{P}}^{-1} \operatorname{deg}\left(X_{i} ; \delta\right)
$$


Démonstration - On considère le plongement mixte introduit au $\S 5$

$$
\begin{array}{cccc}
\sigma: & \boldsymbol{P} & \longrightarrow & \boldsymbol{P}_{n} \\
\left(\boldsymbol{x}_{1}, \ldots, \boldsymbol{x}_{q}\right) & \longmapsto & \left(\ldots: \prod_{i=1}^{q} \prod_{j=1}^{n_{i}} x_{i, j}^{\alpha_{i, j}}: \ldots\right)_{\substack{\alpha_{i} \in N^{n_{i}} \\
\left|\alpha_{i}\right|=\delta_{i}}, i=0, \ldots, q}
\end{array}
$$

pour se ramener au théorème 1. D'après le corollaire 9 on a $H_{g}(\sigma(Y) ; 1)=H_{g}(Y ; \delta) \geq$ $2 c_{\boldsymbol{P}}^{-1} \operatorname{deg}(Y ; \delta)=2 c_{\boldsymbol{P}}^{-1} \operatorname{deg}(\sigma(Y))$ pour toute variété $Y \subset X_{0}$ incomplètement définie sur $\boldsymbol{k}$ dans $X_{0}$ (donc dans $\boldsymbol{P}$ ) par des formes de degré $\leq \delta$. De plus,

$$
\operatorname{Ord}_{\sigma(\Phi)}\left(\sigma\left(X_{0}\right)\right)=\operatorname{Ord}_{\Phi}\left(X_{0}\right)>c_{\boldsymbol{P}}^{-1} \operatorname{deg}\left(X_{0} ; \delta\right)=c_{\boldsymbol{P}}^{-1} \operatorname{deg}\left(\sigma\left(X_{0}\right)\right)
$$

(car $\left.f_{\sigma\left(X_{0}\right)}=f_{X_{0}}\right)$. On vérifie donc que $\sigma\left(X_{0}\right)$ satisfait les hypothèses i) et ii) de la proposition 6. Celle-ci (avec $\delta=1$ et $c=c_{\boldsymbol{P}}$ ) entraîne (comme dans la démonstration du théorème 1 , voir $\S 4$ ) l'existence pour tout $i=0, \ldots, d_{0}-1$ d'une variété $Z$ de dimension $d_{0}-i$, contenue dans $\sigma\left(X_{0}\right)$ et satisfaisant $\operatorname{deg}(Z) \leq \operatorname{deg}\left(\sigma\left(X_{0}\right)\right)$ et $\operatorname{Ord}_{\sigma(\Phi)}(Z)>c_{\boldsymbol{P}}^{-1} \operatorname{deg}(Z)$. En particulier, cette variété s'écrit $Z=\sigma\left(X_{i}\right)$ avec $X_{i} \subset X_{0}$ et $X_{i}$ satisfait $\operatorname{deg}\left(X_{i} ; \delta\right)=\operatorname{deg}(Z) \leq \operatorname{deg}\left(\sigma\left(X_{0}\right)\right)=\operatorname{deg}\left(X_{0} ; \delta\right)$ et

$$
\operatorname{Ord}_{\Phi}\left(X_{i}\right)=\operatorname{Ord}_{\sigma(\Phi)}(Z)>c_{\boldsymbol{P}}^{-1} \operatorname{deg}(Z)=c_{\boldsymbol{P}}^{-1} \operatorname{deg}\left(X_{i} ; \delta\right) \text {. }
$$

On applique cela à $\tilde{\boldsymbol{P}}:=\boldsymbol{P}_{1} \times \boldsymbol{P}$ avec $\tilde{\Phi}:=((1: z), \Phi)$ et le multidegré $(\eta, \delta)$ avec $\min \left(\delta_{1} ; \ldots ; \delta_{q}\right) \geq c_{\tilde{\boldsymbol{P}}}:=2(n+2)^{n+2}$. Soit $\tilde{X}_{0} \subset \tilde{\boldsymbol{P}}$ une variété de dimension $d_{0}+1$ satisfaisant $\operatorname{Ord}_{\tilde{\Phi}}\left(\tilde{X}_{0}\right)>c_{\tilde{\boldsymbol{P}}}^{-1} \operatorname{deg}\left(\tilde{X}_{0} ;(\eta, \delta)\right)$. On déduit du théorème 10 l'existence de variétés $\tilde{X}_{i} \subset \tilde{\boldsymbol{P}}(\boldsymbol{k}), i=1, \ldots, d_{0}$, de dimension $d_{0}+1-i$ et définies sur $\boldsymbol{k}$, satisfaisant

$$
\operatorname{deg}\left(\tilde{X}_{i} ;(\eta, \delta)\right) \leq \operatorname{deg}\left(\tilde{X}_{0} ;(\eta, \delta)\right)
$$

et

$$
\operatorname{Ord}_{\tilde{\Phi}}\left(\tilde{X}_{i}\right)>c_{\tilde{\boldsymbol{P}}}^{-1} \operatorname{deg}\left(\tilde{X}_{i} ;(\eta, \delta)\right) .
$$

On intersecte $\tilde{X}_{d_{0}}$, qui est de dimension 1 , avec l'hyperplan $H:=\left\{x_{0} z-x_{1}=0\right\} \times \boldsymbol{P}$, on ne peut avoir $\tilde{X}_{d_{0}} \subset H$ car $\tilde{X}_{d_{0}}$ est définie sur $\boldsymbol{k}$. De plus, si $\tilde{X}_{d_{0}}$ est de la forme $\{u\} \times Y$ pour une certaine courbe $Y \subset \boldsymbol{P}(\boldsymbol{k})$ et $u \in \boldsymbol{P}_{1}(\boldsymbol{k})$, alors on vérifie

$$
c_{\tilde{\boldsymbol{P}}}^{-1} \operatorname{deg}(Y ; \delta)=c_{\tilde{\boldsymbol{P}}}^{-1} \operatorname{deg}\left(\tilde{X}_{d_{0}} ;(\eta, \delta)\right)<\operatorname{Ord}_{\tilde{\Phi}}\left(\tilde{X}_{d_{0}}\right) \leq \operatorname{Ord}_{(1: z)}(\{u\}) \operatorname{deg}(Y ; \mathbf{1}) .
$$

Ainsi, $\tilde{X}_{d_{0}}$ n'est pas de la forme $\{u\} \times Y$ avec $Y \subset \boldsymbol{P}(\boldsymbol{k})$ et $u \in \boldsymbol{P}_{1}(\boldsymbol{k})$, et donc le cycle $Z:=\tilde{X}_{d_{0}} \cap H$ est non vide, de dimension 0 , défini sur $\boldsymbol{k}(z)$ tel que

$$
\begin{gathered}
\operatorname{deg}(Z)=d_{(1,0)}\left(\tilde{X}_{d_{0}}\right) \\
h(Z ; \delta)=\sum_{i=1}^{q} \delta_{i} d_{\left(0, \varepsilon_{i}\right)}\left(\tilde{X}_{d_{0}}\right) \\
h(Z ; \delta)+\eta \operatorname{deg}(Z)=\operatorname{deg}\left(\tilde{X}_{d_{0}} ;(\eta, \delta)\right) \leq \operatorname{deg}\left(\tilde{X}_{0} ;(\eta, \delta)\right)
\end{gathered}
$$

et

$$
\begin{aligned}
\sum_{\alpha \in Z} \min _{1 \leq i \leq q}\left(\operatorname{Ord}_{z}\left(\alpha_{i} \wedge \phi_{i}\right)\right)=\operatorname{Ord}_{\Phi}(Z) & \geq \operatorname{Ord}_{\tilde{\Phi}}\left(\tilde{X}_{d_{0}}\right) \\
& \geq c_{\tilde{\boldsymbol{P}}}^{-1} \operatorname{deg}\left(\tilde{X}_{0} ;(\eta, \delta)\right) \\
& \geq c_{\tilde{\boldsymbol{P}}}^{-1}(h(Z ; \delta)+\eta \operatorname{deg}(Z))
\end{aligned}
$$

On a ainsi établit le corollaire suivant : 
Corollaire 11 - Sous les hypothèses du théorème 10 soit $\eta \in \boldsymbol{N}^{*}, \delta \in\left(\boldsymbol{N}^{*}\right)^{q}$ tel que $\min \left(\delta_{1} ; \ldots ; \delta_{q}\right) \geq c_{\boldsymbol{P}_{1} \times \boldsymbol{P}}$, on suppose que $\tilde{X}_{0} \subset \boldsymbol{P}_{1} \times \boldsymbol{P}$ est définie sur $\boldsymbol{k}$ dans $\boldsymbol{P}_{1} \times \boldsymbol{P}$ par des formes de multidegré $\leq(\eta, \delta)$ et satisfait

$$
\operatorname{Ord}_{\tilde{\Phi}}\left(\tilde{X}_{0}\right)>c_{\boldsymbol{P}_{1} \times \boldsymbol{P}}^{-1} \operatorname{deg}\left(\tilde{X}_{0} ;(\eta, \delta)\right)
$$

Alors, il existe un cycle $Z \subset \tilde{X}_{0}$ de dimension 0 et défini sur $\boldsymbol{k}(z)$ tel que $\left.h(Z ; \delta)+\eta \operatorname{deg}(Z) \leq \operatorname{deg}\left(\tilde{X}_{0} ; \eta, \delta\right)\right)$ et

$$
\sum_{\alpha \in Z} \min _{1 \leq i \leq q}\left(\operatorname{Ord}_{0}\left(\alpha_{i} \wedge \phi_{i}\right)\right)>c_{\boldsymbol{P}_{1} \times \boldsymbol{P}}^{-1}(h(Z ; \delta)+\eta \operatorname{deg}(Z)) .
$$

En particulier, si $\tilde{X}_{0}$ est de codimension 1 le corollaire 11 permet d'établir :

Lemme de transfert multiprojectif - Soit $b \geq 2^{n-1}, \mu, \mu_{1}, \ldots, \mu_{q} \geq 1$ et $\Phi \in \boldsymbol{P}(C)$, si une forme $P \in A[z]$ satisfait $d_{z}^{\circ} P, d_{\boldsymbol{x}_{1}}^{\circ} P, \ldots, d_{\boldsymbol{x}_{q}}^{\circ} P \geq c_{\tilde{\boldsymbol{P}}}$ et

$$
\operatorname{ord}_{z}(P \circ \Phi)-\sum_{i=1}^{q} d_{\boldsymbol{x}_{i}}^{\circ} P . \operatorname{ord}_{z}\left(\phi_{i}\right)-d_{z}^{\circ}(P)>b\left(d_{z}^{\circ} P\right)^{\mu} \cdot\left(d_{\boldsymbol{x}_{1}}^{\circ} P\right)^{\mu_{1} n_{1}} \ldots\left(d_{\boldsymbol{x}_{q}}^{\circ} P\right)^{\mu_{q} n_{q}}
$$

alors il existe un cycle $Z \subset \boldsymbol{P}_{n}(\overline{\boldsymbol{k}(z)})$ de dimension 0 et défini sur $\boldsymbol{k}(z)$, contenu dans le lieu des zéros de $P$, satisfaisant

$$
\operatorname{deg}(Z) d_{z}^{\circ} P+\sum_{i=1}^{q} h_{i}(Z) d_{\boldsymbol{x}_{i}}^{\circ} P \leq c_{\tilde{\boldsymbol{P}}} b^{\frac{n-1}{n}}\left(d_{z}^{\circ} P\right)^{\mu \frac{n-1}{n}+\frac{1}{n}} \cdot \prod_{j=1}^{q}\left(d_{\boldsymbol{x}_{j}}^{\circ} P\right)^{\mu_{j} n_{j} \frac{n-1}{n}+\frac{n_{j}}{n}}
$$

et, en notant $\langle\boldsymbol{\mu}, \boldsymbol{n}\rangle:=\mu+\mu_{1} n_{1}+\ldots+\mu_{q} n_{q}$

$$
\sum_{\alpha \in Z} \min _{1 \leq i \leq q}\left(\operatorname{Ord}_{z}(\alpha \wedge \phi)\right)>c_{\tilde{\boldsymbol{P}}}^{-1}\left(\tilde{b} \operatorname{deg}(Z)^{\langle\boldsymbol{\mu}, \boldsymbol{n}\rangle-\mu} \cdot \prod_{i=1}^{q} h_{i}(Z)^{\left(\langle\boldsymbol{\mu}, \boldsymbol{n}\rangle-\mu_{i}\right) n_{i}}\right)^{\frac{1}{\langle\boldsymbol{\mu}, \boldsymbol{n}\rangle(n-1)+1}}
$$

où on a posé $\tilde{b}:=c_{\tilde{\boldsymbol{P}}}\left(\frac{n_{1} ! \ldots n_{q} !}{(n+1) !}\right)^{\langle\boldsymbol{\mu}, \boldsymbol{n}\rangle} b$.

Démonstration - Posons $b_{1}:=c_{\tilde{\boldsymbol{P}}} \frac{n_{1} ! \ldots n_{q} !}{(n+1) !} b$, on applique le corollaire 11 avec $\tilde{X}_{0}=\mathcal{Z}(P)$ et

$$
\left\{\begin{array}{l}
\eta=b_{1}^{1 / n}\left(d_{z}^{\circ} P\right)^{1+\frac{\mu-1}{n}} \cdot \prod_{j=1}^{q}\left(d_{\boldsymbol{x}_{j}}^{\circ} P\right)^{\left(\mu_{j}-1\right) \frac{n_{j}}{n}} \\
\delta_{i}=b_{1}^{1 / n} d_{\boldsymbol{x}_{i}}^{\circ} P \cdot\left(d_{z}^{\circ} P\right)^{\frac{\mu-1}{n}} \cdot \prod_{j=1}^{q}\left(d_{\boldsymbol{x}_{j}}^{\circ} P\right)^{\left(\mu_{j}-1\right) \frac{n_{j}}{n}}, \quad i=1, \ldots, q .
\end{array}\right.
$$

On vérifie $\operatorname{deg}\left(\tilde{X}_{0} ;(\eta, \delta)\right)=\frac{(n+1) !}{n_{1} ! \ldots n_{q} !} b_{1}\left(d_{z}^{\circ} P\right)^{\mu} \cdot \prod_{i=1}^{q}\left(d_{\boldsymbol{x}_{i}}^{\circ} P\right)^{\mu_{i} n_{i}}$ et l'hypothèse faite sur $P$ montre que la condition du corollaire 11: $\operatorname{Ord}_{\Phi}\left(\tilde{X}_{0}\right)>c_{\tilde{\boldsymbol{P}}}^{-1} \operatorname{deg}\left(\tilde{X}_{0} ;(\eta, \delta)\right)$, est 
satisfaite, car le membre de gauche est précisément $\operatorname{Ord}_{\Phi}\left(\tilde{X}_{0}\right)$. Le corollaire nous fournit donc un cycle $Z \subset \boldsymbol{P}(\overline{\boldsymbol{k}})$ de dimension 0 , défini sur $\boldsymbol{k}(z)$, contenu dans $\tilde{X}_{0}=\mathcal{Z}(P)$ tel que :

$$
\left\{\begin{aligned}
\operatorname{deg}(Z) & \leq \frac{(n+1) !}{n_{1} ! \ldots n_{q} !} b_{1}^{\frac{n-1}{n}}\left(d_{z}^{\circ} P\right)^{(\mu-1) \frac{n-1}{n}+1} \cdot \prod_{j=1}^{q}\left(d_{\boldsymbol{x}_{j}}^{\circ} P\right)^{\left(\mu_{j}-1\right) n_{j} \frac{n-1}{n}+n_{j}} / d_{z}^{\circ} P \\
h_{i}(Z) & \leq \frac{(n+1) !}{n_{1} ! \ldots n_{q} !} b_{1}^{\frac{n-1}{n}}\left(d_{z}^{\circ} P\right)^{(\mu-1) \frac{n-1}{n}+1} \cdot \prod_{j=1}^{q}\left(d_{\boldsymbol{x}_{j}}^{\circ} P\right)^{\left(\mu_{j}-1\right) n_{j} \frac{n-1}{n}+n_{j}} / d_{\boldsymbol{x}_{i}}^{\circ} P
\end{aligned}\right.
$$

et

$$
\sum_{\alpha \in Z} \min _{1 \leq i \leq q}\left(\operatorname{Ord}_{0}\left(\alpha_{i} \wedge \phi_{i}\right)\right)>c_{\tilde{\boldsymbol{P}}}^{-1}\left(\operatorname{deg}(Z) \eta+\sum_{i=1}^{q} h_{i}(Z) \delta_{i}\right)
$$

On déduit de (4)

$$
\max _{1 \leq i \leq q}\left(\operatorname{deg}(Z) \eta ; h_{i}(Z) \delta_{i}\right) \geq\left(\tilde{b} \operatorname{deg}(Z)^{\langle\boldsymbol{\mu}, \boldsymbol{n}\rangle-\mu} \prod_{j=1}^{q} h_{j}(Z)^{\left(\langle\boldsymbol{\mu}, \boldsymbol{n}\rangle-\mu_{j}\right) n_{j}}\right)^{\frac{1}{\langle\boldsymbol{\mu}, \boldsymbol{n}\rangle(n-1)+1}}
$$

et enfin la minoration voulue en combinant avec (5).

Remarque - Dans la démonstration ci-dessus nous avons choisi les paramètres $\eta$, $\delta_{1}, \ldots, \delta_{q}$ optimaux en leur imposant d'être proportionnels à $d_{z}^{\circ} P, d_{\boldsymbol{x}_{1}}^{\circ} P, \ldots, d_{\boldsymbol{x}_{q}}^{\circ} P$ respectivement. D'autres choix sont envisageables qui conduisent à des conclusions différentes du lemme de transfert.

\section{Références}

[1] Bugeaud Y. - On the approximation by algebraic numbers with bounded degree, dans Algebraic Number Theory and Diophantine Analysis, Graz, 1998, F. HalterKoch \& R.F. Trichy éds, Walter de Gruyter, 2000, 47-53.

[2] Bugeaud Y. - Approximation par des nombres algébriques, J. Number Theory, 84-1, 2000, 15-33.

[3] Chardin M. - Une majoration de la fonction de Hilbert et ses conséquences pour l'interpolation algébrique, Bull. Soc. Math. France, 117, 1989, 305-318.

[4] Chardin M. \& Philippon P. - Régularité et interpolation, J. Algebraic Geom., 8, 1999, 471-481; Erratum, ibidem, 11, 2002, 599-600. 
[5] Chudnovsky G.V. - Contribution to the theory of transcendental numbers, Math. Surveys Monogr., 19, Amer. Math. Soc., 1984.

[6] Diaz G. - Une nouvelle propriété d'approximation diophantienne, C.R. Acad. Sci. Sér. I, 324, 1997, 969-972.

[7] Durand A. - Approximations algébriques d'un nombre transcendant, dans Cinquante ans de polynômes, Lecture Notes in Math. 1415, Springer, 1990, 94-96.

[8] Goto S. \& Watanabe K. - On graded rings I, J. Math. Soc. Japan, 30/2, 1978, 179-213.

[9] Jabbouri E.M. - Sur un critère pour l'indépendance algébrique de P. Philippon, dans Approximation Diophantienne et Nombres Transcendants, Luminy 1990, Walter de Gruyter, 1995, 195-202.

[10] Jadot C. - Critères pour l'indépendance algébrique et linéaire, Thèse, Univ. P. \& M. Curie, 1996.

[11] Laurent M. - New methods in algebraic independence, dans Number Theory. Diophantine, computational and algebraic aspects, Eger, 29 July - 2 August 1996, K. Györy, A. Pethö \& V. Sós éds, Walter de Gruyter, Berlin, 1998, 311-330.

[12] Laurent M. - Diophantine approximation and algebraic independence, dans Colloque Franco-Japonais, Théorie des nombres transcendants, Sem. Math. Sci., 27, Keio Univ., 1999, 75-89.

[13] Laurent M. - Some remarks on the approximation of complex numbers by algebraic numbers, dans Proceedings of the 2nd Panhellenic Conference in Algebra and Number Theory, 13-14 June 1998, Bull. Greek Math. Soc., 42, 1999, 49-57.

[14] Laurent M. \& Roy D. - Sur l'approximation algébrique en degré de transcendance un, Ann. Inst. Fourier (Grenoble), 49-1, 1999, 27-55.

[15] Laurent M. \& Roy D. - Criteria of algebraic independence with multiplicities and interpolation determinants, Trans. Amer. Math. Soc., 351-5, 1999, 1845-1870.

[16] Laurent M. \& Roy D. - Criteria of algebraic independence with multiplicities and approximation by hypersurfaces, J. Reine Angew. Math., 536, 2001, 65-114.

[17] Nesterenko Y.V. \& Philippon P., Introduction to algebraic independence theory, Lecture Notes in Math., 1752, Springer, 2001.

[18] Nishioka K., Mahler functions and transcendence, Lecture Notes in Math., 1631, Springer, 1996. 
[19] Philippon P. - Critère pour l'indépendance algébrique, Inst. Hautes Études Sci. Publ. Math., 64, 1986, 5-52.

[20] Philippon P. - Une approche méthodique pour la transcendance et l'indépendance algébrique de valeurs de fonctions analytiques, J. Number Theory, 64, 1997.

[21] Philippon P. - Some remarks on proofs of algebraic independence, Diophantine geometry \& Criteria for algebraic independence, chap. 4, 6 \& 8 dans Introduction to algebraic independence theory, Y.V. Nesterenko \& P. Philippon éds, Lecture Notes in Math. 1752, Springer, 2001, 47-51, 83-94 \& 133-141.

[22] Philippon P. - Approximations algébriques des points dans les espaces projectifs I, J. Number Theory, 81, 2000, 234-253.

[23] Roy D. - Approximation algébrique simultanée de nombres de Liouville, Canadian Math. Bull., 44-1, 2001, 115-120.

[24] Roy D. \& Waldschmidt M. - Approximation diophantienne et indépendance algébrique de logarithmes, Ann. Sci. École Norm. Sup. (4), 30, 1997, 753-796.

[25] Roy D. \& Waldschmidt M. - Simultaneous approximation and algebraic independence, The Ramanujan J., 1, 1997, 379-430.

[26] Waldschmidt M. - From simultaneous approximation to algebraic independence, dans Summer School on Number Theory and its applications, Bilkent, Ankara, 1996, Cem Y. Yıldırım \& S. Stepanov éds, Lecture Notes in Pure Appl. Math., 204, Marcel Dekker Inc., 283-305.

[27] Waldschmidt M. - Transcendence and algebraic independence of Liouville-like numbers, dans Proceedings of the 2nd Panhellenic Conference in Algebra and Number Theory, 13-14 June 1998, Bull. Greek Math. Soc., 42, 1999, 119-134.

[28] Waldschmidt M. - Algebraic independence of transcendental numbers : a survey, dans Number Theory, R.P. Bambah, V.C. Dumir \& R.J. Hans Gill éds, Hindustan Book Agency, New Delhi \& Indian National Sci. Acad., 1999, 497-527.

[29] Waldschmidt M. - Conjectures for large transcendence degree, dans Algebraic Number Theory and Diophantine Analysis, Graz, 1998, F. Halter-Koch \& R.F. Trichy éds, Walter de Gruyter, 2000, 497-520.

[30] Waldschmidt M. - Open diophantine problems, Moscow Math. J., 4, 2004, 245-305.

[31] Waldschmidt M. - Diophantine approximation on linear algebraic groups. Transcendence properties of the exponential function in sevral variables, Grund. Math. Wiss., 326, Springer, 2000, 633p. 
APproximations FONCTIONNELLES DES COURBES DES ESPACES PROJECTIFS

[32] Wirsing E. - Approximation mit algebraischen Zahlen beschränkten Grades, J. Reine Angew. Math., 206, 1961, 67-77.

[33] Zorin E. - Lemmes de multiplicités et applications, thèse Univ. Pierre et Marie Curie, 2010. 